\title{
Automatic Detection of Kiwifruit Defects Based on Near-Infrared Light Source*
}

\author{
Pingping Li, Yongjie Cui ${ }^{* *}$, Yufeng Tian, Fanian Zhang, Xiaxia Wang, and Shuai Su \\ College of Mechanical and Electronic Engineering, Northwest A\&F University, \\ Yangling Shaanxi, 712100, P.R. China \\ \{lipingping, cuiyongjie, tianyufeng, zhangfanian, \\ wangxiaxia, sushuai\}@nwsuaf.edu.cn
}

\begin{abstract}
A mathematical model that expresses the relationship between Near-infrared light intensity and automatic threshold for automatic kiwifruit surface defect detection was established. By applying different levels of Near-infrared light intensity to machine vision system, 268 images were collected. Then the images were processed with MATLAB using the method to detect kiwifruit defects based on Near-infrared light source .The obtained 268 sets of data on Automatic Threshold $T_{0}$ and Manual Threshold $T_{1}$ were divided into 19 groups according to different aperture and light intensity. After processing data, a series of linear equations about the relationship between Near-infrared light intensity and Automatic Threshold $T_{0}$, with function fitting coefficient of $R^{2}>95 \%$ was obtained. Finally, relationship between $T_{0}$ and $T_{1}$ was analyzed according to the effectiveness of image processing results and constant $P$ was introduced to revise Automatic Threshold $T_{0}$. Thus, a mathematical model needed to gain kiwifruit defects detection threshold, namely Model Threshold $T$, was established.
\end{abstract}

Keywords: Image processing, Near-infrared light intensity, Automatic threshold, Manual threshold, Model threshold, Linear relationship.

\section{Introduction}

The kiwifruit, or often shortened to kiwi in many parts of the world, is the edible berry of a cultivar group of the woody vine Actinidia deliciosa and hybrids between this and other species in the genus Actinidia. It's nutritious and has high medicinal value [1]. China is one of the major areas in producing kiwi. Currently, kiwifruit postharvest sorting processing is still performed manually and its surface defects judgment depends on human completely. Standard Sphere Method [2-5] which uses machine vision technology to separate the fruit surface defects has achieved good results. However, with the traditional RGB [6][7], CCD imaging systems [8-11], the angle between the camera and edge light reflection direction of sphere and ellipsoid fruits remains very large. According

\footnotetext{
Foundation item: The Project-sponsored by SRF for ROCS, SEM (KS08021101), Project supported by the National Natural Science Foundation of China (61175099), Northwest Agriculture and Forestry University Talent Fund (Z111020902).

** Corresponding author.
}

D. Li and Y. Chen (Eds.): CCTA 2012, Part I, IFIP AICT 392, pp. 189-198, 2013.

(C) IFIP International Federation for Information Processing 2013 
to Lambertian light laws of reflection, the fruit edge and surface defects both have lower gray level. Therefore it is difficult to detect and distinguish the defect. In addition, there is a challenge in uniform illumination with the RGB and CCD systems. To overcome these difficulties, near-infrared hyper spectral is used by many research institutes to test external qualities of agriculture products such as maturity of strawberry [12], bruises of strawberry [13] [14], apple [15] and chestnut [16] defects etc.

There are two assumptions in Standard Sphere Method. First assumption is that the shape of fruit is either standard sphere or ellipsoid. Second assumption is that light illumination in machine vision field is uniform. Under these conditions, optical system consisting of lens and camera can be a linear system [17]. In the case of kiwifruit, its shape is quasi-ellipsoid. With the Near-infrared light source, the issue of fruit surface reflective area can be overcome as it plays the role of uniform illumination according to preliminary studies [18][19]. This paper aims at collecting images of defective kiwifruit with different light intensity by adjusting the Near-infrared light intensity, then processing images by detection methods for kiwifruit surface defects grading, and finally analyzing the relationship between binarization threshold of kiwifruit defects and Near-infrared light intensity, to establish a mathematical model.

\section{Material and Method}

\subsection{Test Materials and Equipment}

Test kiwifruit samples were "Qin Mei" cultivars. They were bought from Xizhai Village, Qinghua Country, Mei County, Shaanxi Province. All of the fruits (total 182 samples) were picked up on site and kept in cartons. Weight of a single fruit ranged from 69.6g to 224.0g. Tests were carried out at College of Mechanical and Electronic Engineering, Northwest A\&F University.

Firstly the fruits were classified; according to the type of defects such as sunburn, parasitic spot. Then intact fruits were scratched to make them defective.

In terms of equipment, lamp house was made of black organic plastic material to avoid external light interference. The camera used was DALSA CCD camera (matching dedicated image acquisition and debugging software Sapera Cam Expert) and the lens was Camera FUJINON HF16HA-1B (aperture range 1.4 16.0). PC used to save data after image processing was Lenovo ThinkPad E420. LFX2-100IR850 Near-infrared light source and dedicated power supply PD-3024-K1320-014 with Light source extension line $(1 \mathrm{~m}, 24 \mathrm{~V})$ also consisted the test equipment. As a background a white soleplate was provided to enhance the contrast of the background and kiwifruit. The distance between camera FUJINON HF16HA-1B and kiwifruit was $370 \mathrm{~mm}$ and distance between Near-infrared light source and kiwifruit was $110 \mathrm{~mm}$, as shown in Fig. 1.

LFX2-100IR850 Near-infrared light source needed to be attached to the light source dedicated power supply CCS PD-3024- K1320-014 with a light source extension line $(1 \mathrm{~m}, 24 \mathrm{~V})$.There are 16 brightness coarse adjustment gears and 16 fine adjustment gears respectively. Fig.1 shows pictures of Near-infrared plane illuminator LFX2-100IR850 and light source dedicated power supply CCS PD-3024- K1320-014. The light source needed to be warmed up for 30 minutes before usage. 


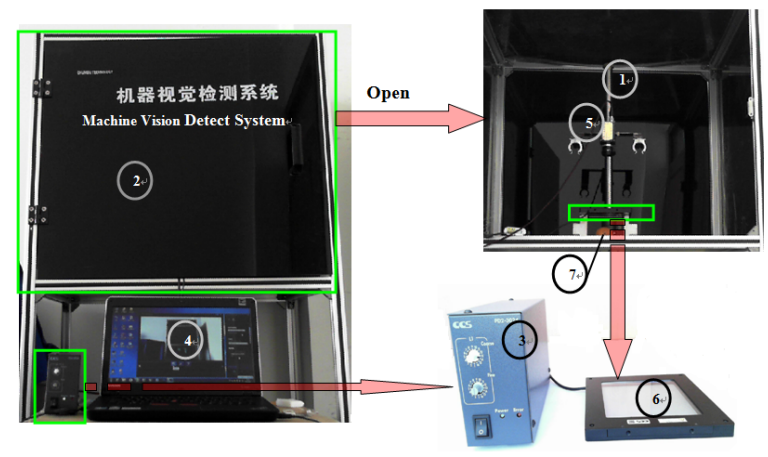

1. Lifting frame 2. Lamp house 3. Light intensity regulator 4. PC 5.CCD Camera 6.Near-infrared light source

$$
\text { 7. Kiwifruit }
$$

Fig. 1. Kiwifruit Machine Vision Detect System

\subsection{Research Program}

As shown in Fig.2, the left dotted line box shows the image processing of kiwifruit defects detection [18]. Although this method is able to extract the fruit surface defects, there are two problems exist:

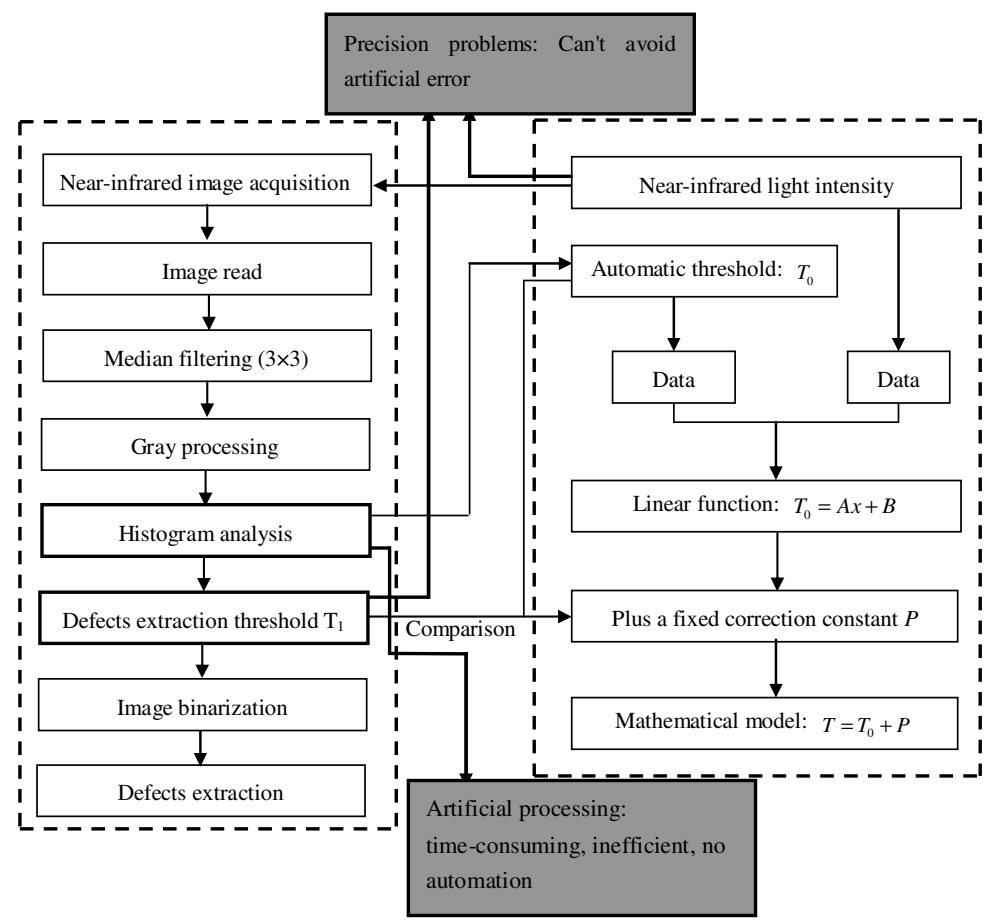

Fig. 2. The Block Diagram of Research 
Problem 1: It's time-consuming to extract the image parameters of fruit surface defects manually and the automatic detection of fruit defects is a mainstream in the development of nondestructive testing of the fruits.

Problem 2: As shown in Fig.2, the automatic defection threshold $T_{0}$ will produce error and it will influence the accuracy of fruit defection.

To extract the fruit defects threshold automatically, this paper proposed a method based on Near-infrared light intensity with Automatic Threshold $T_{0}$ and thus established a mathematical model. The dotted line box on the right is the solution diagram. As $T_{1}$ obtained by manual analysis will produce error. Therefore, Automatic Threshold $T_{0}$ was introduced to solve the problem, where $T_{0}$ is automatically generated by the image processing program and it reduces the systematic errors effectively. $T_{0}$ cannot be used to detect defect directly. After comparing $T_{1}$ with $T_{0}$, a correction constant $P$ was introduced to revise $T$, as $T=T_{0}+P$.

\subsection{Test Methods and Procedures}

Scratch wound is one of kiwifruit surface defects which is the most difficult defect to be extracted. So we took kiwifruit with scratch wound as objects to establish the relationship between Near-infrared light intensity and scratch defects and to determine the automatic threshold of surface defects.

Table 1. Test conditions and image collection

\begin{tabular}{cccc}
\hline $\begin{array}{c}\text { Aperture } \\
(1.4 \sim 16.0)\end{array}$ & $\begin{array}{c}\text { Coarse adjustment } \\
(1 \sim 16)\end{array}$ & $\begin{array}{c}\text { Fine adjustment } \\
(1 \sim 16)\end{array}$ & Image quantity \\
\hline 1.4 & 1 & $3 \sim 11$ & 9 \\
3.7 & $2 、 3$ & $1 \sim 16$ & 32 \\
4.0 & $3 、 4$ & $1 \sim 16$ & 32 \\
6.0 & $5 \sim 10$ & $1 \sim 16$ & 96 \\
8.0 & 5 & $1 \sim 16$ & 33 \\
8.0 & $6 \sim 16$ & $1,9 、 16$ & 32 \\
12.0 & $6 \sim 16$ & $1,9 、 16$ & 16 \\
16.0 & $8 \sim 16$ & 1,16 & 268 \\
\hline
\end{tabular}




\section{Test Procedures}

(1) Put the scratch kiwifruits into lamp house; adjust the camera aperture according to Tab.1. Adjust the brightness coarse adjustment gears and fine adjustment gears in accordance with Tab.1. Finally collect images using supporting software Sapera Cam Expert and adjust R, G, B to $0 \mathrm{~dB}$.

(2) Number the collected images and light intensity record mode. E.g. coarse adjustment gear 1 and fine adjustment 1 16 are recorded as 101,102,103, 104...116, while coarse adjustment gear 1 16 and fine adjustment 1 are recorded as 1601, 1602, 1603, 1604...1616.

(3) Process the numbered images using the method described in [18], and record $T_{0}, T_{1}$ and image processing results.Tab. 2 is part of the test data records.

(4) Change the Near-infrared light intensity from 103 to 111 , gain $T_{0}$ using the method as mentioned in Step (3), and adjust the threshold interval to gain $T_{1}$. It is obtained by adding a constant $P$ to $T_{0}$, as shown in Tab.4.

(5) Finally, analyze and process the data with Microsoft Excel. Although the final threshold used in image processing is defect detection threshold, the mathematic model expresses the relationship between $T_{0}$ and Near-infrared intensity. The reason is that $T_{1}$ is a manual selected threshold according to the effectiveness of image processing results. If $T_{l}$ is selected to analyze data, it will increase the error. Thus $T_{0}$ obtained by MATLAB is used to establish the exact relationship between threshold and light intensity. Then analyze the relationship between $T_{1}$ and $T_{0}$. Finally introduce a correction constant $P$ to revise $T_{0}$.

Table 2. Part of the test data

\begin{tabular}{ccccc}
\hline No. & Aperture & Light intensity & Automatic Threshold $T_{0}$ & Manual Threshold $T_{1}$ \\
\hline 001 & 1.4 & 103 & 0.0941 & 0.1400 \\
002 & 1.4 & 104 & 0.1333 & 0.2400 \\
003 & 1.4 & 105 & 0.1686 & 0.2590 \\
004 & 1.4 & 106 & 0.2039 & 0.3590 \\
005 & 1.4 & 107 & 0.2353 & 0.4000 \\
006 & 1.4 & 108 & 0.2706 & 0.4400 \\
007 & 1.4 & 109 & 0.3020 & 0.5300 \\
008 & 1.4 & 110 & 0.3333 & 0.5533 \\
009 & 1.4 & 111 & 0.3686 & 0.6700 \\
\hline
\end{tabular}




\section{Results and Discussion}

\subsection{Mathematical Model}

The data were analyzed using Microsoft Excel, and the data in Tab.2 were used to produce the function graph in Fig.3. The intensity of Near-infrared was from 103 to 111 , and the automatic threshold interval was from 0.0900 to 0.4000 . Fig. 3 -a is the linear diagram of $T_{0}$ and light intensity, in Tab.3, we established the equation $T_{0}=0.0339 x-3.3884, R^{2}=0.9992$. Fig. $3-\mathrm{b}$ is the linear diagram of $T_{1}$ and the light intensity and we can find out the equation $T_{l}=0.0614 x-6.1688, R^{2}=0.9844$.

The function fitting degree in Fig.3-a is higher than that in Fig.3-b. This is because the $T_{0}$ is free from the human error of $T_{l}$.

Similarly, we processed the 19 groups' data and the results were recorded inTab.3.

As a result, the function relation graphs between Near-infrared intensity and $T_{0}$ was gained. It was linear. All of the 268 groups of data were processed by this method, the results were shown in Tab.3, with $R^{2}>95 \%$. Thus the relation between Near-infrared intensity and $T_{0}$ can be defined as follows:

$$
T_{0}=A x+B \text {. }
$$

Where: $T_{0}, x, \mathrm{~A}, \mathrm{~B}$ stand for automatic threshold, Near-infrared intensity, light intensity coefficient, Near- infrared fine adjustment influence coefficient, respectively.

(1) In Tab.3, the functions from 01 to 11 were gained under the same aperture and light intensity with both brightness coarse and variable fine adjustment. They showed linear relationship. In addition, value $A$ and $B$ are variables. Value $A$ increased as the light intensity enhanced, while value $B$ had the opposite trend.

(1) The functions from 12 to 19 were gained under the same aperture and light intensity with brightness fine adjustment and variable coarse adjustment. They also showed linear relationship. In addition, value $A$ didn't change as the light intensity enhanced, while value $B$ increased with light intensity enhancing.

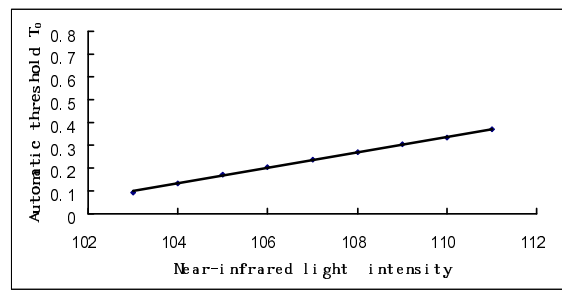

$\mathbf{a}$

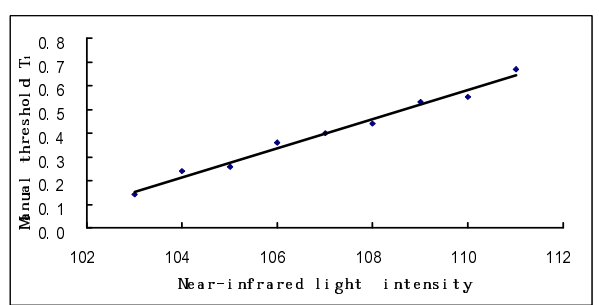

b

Fig. 3. The relation between light intensity and $T_{0} 、 T_{l}$ (Data in Tab. 2)

(2) Considering that $T_{0}$ was not used to detect the fruit surface defects directly, it was necessary to define the correction constant $P$ value. The relationship between Near-infrared intensity and defection threshold was defined as follows: 


$$
T=T_{0}+P .
$$

This is because the $T_{0}$ is free from the human error of $T_{l}$.

Where: $T, T_{0}, P$ stand for defects threshold, automatic threshold, threshold correct constant, respectively.

Table 3. The relation between light intensity and automatic threshold

\begin{tabular}{|c|c|c|c|c|c|}
\hline No. & Aperture & $\begin{array}{c}\text { Coarse } \\
\text { adjustment }\end{array}$ & $\begin{array}{c}\text { Fine } \\
\text { adjustment }\end{array}$ & $\begin{array}{c}\text { Function } \\
\left(T_{0}=\mathrm{A} x+B\right)\end{array}$ & $R^{2}$ \\
\hline 01 & 1.4 & 1 & $3 \sim 11$ & $T_{0}=0.0339 x-3.3884$ & 0.9992 \\
\hline 02 & 3.7 & 2 & $1 \sim 16$ & $T_{0}=0.0057 x-1.0295$ & 0.9975 \\
\hline 03 & 3.7 & 3 & $1 \sim 16$ & $T_{0}=0.0063 x-1.7055$ & 0.9985 \\
\hline 03 & 4.0 & 3 & $1 \sim 16$ & $T_{0}=0.0050 x-1.3321$ & 0.9976 \\
\hline 04 & 4.0 & 4 & $1 \sim 16$ & $T_{0}=0.0052 x-1.8516$ & 0.9935 \\
\hline 05 & 6.0 & 5 & $1 \sim 16$ & $T_{0}=0.0027 x-1.1500$ & 0.9882 \\
\hline 06 & 6.0 & 6 & $1 \sim 16$ & $T_{0}=0.0026 x-1.3268$ & 0.9893 \\
\hline 07 & 6.0 & 7 & $1 \sim 16$ & $T_{0}=0.0027 \mathrm{x}-1.6486$ & 0.9865 \\
\hline 08 & 6.0 & 8 & $1 \sim 16$ & $T_{0}=0.0028 x-1.9274$ & 0.9927 \\
\hline 09 & 6.0 & 9 & $1 \sim 16$ & $T_{0}=0.0029 x-2.2458$ & 0.9930 \\
\hline 10 & 6.0 & 10 & $1 \sim 16$ & $T_{0}=0.0027 x-2.2822$ & 0.9808 \\
\hline 11 & 8.0 & 5 & $1 \sim 16$ & $T_{0}=0.0011 \mathrm{x}-0.4516$ & 0.9545 \\
\hline 12 & 8.0 & $6 \sim 16$ & 1 & $T_{0}=0.0002 x-0.0209$ & 0.9995 \\
\hline 13 & 8.0 & $6 \sim 16$ & 9 & $T_{0}=0.0002 x-0.0109$ & 0.9987 \\
\hline 14 & 8.0 & $6 \sim 16$ & 16 & $T_{0}=0.0002 x-0.0029$ & 0.9993 \\
\hline 15 & 12.0 & $6 \sim 16$ & 1 & $T_{0}=0.0001 x+0.0099$ & 0.9980 \\
\hline 16 & 12.0 & $6 \sim 16$ & 9 & $T_{0}=0.0001 x+0.0129$ & 0.9981 \\
\hline 17 & 12.0 & $6 \sim 16$ & 16 & $T_{0}=0.0001 x+0.0077$ & 0.9929 \\
\hline 18 & 16.0 & $8 \sim 16$ & 1 & $T_{0}=0.00003 x+0.0210$ & 0.9634 \\
\hline 19 & 16.0 & $8 \sim 16$ & 16 & $T_{0}=0.00003 x+0.0291$ & 0.9867 \\
\hline
\end{tabular}


Table 4. The correction constant $P$ for surface defects extraction threshold

\begin{tabular}{cccccc}
\hline$T_{0}$ & $T_{0}<0.1$ & $0.1 \leq T_{0}<0.2$ & $0.2 \leq T_{0}<0.3$ & $0.3 \leq T_{0}<0.4$ & $T_{0} \geq 0.4$ \\
\hline$P$ & 0.0400 & 0.1000 & 0.1600 & 0.2200 & 0.2800 \\
\hline
\end{tabular}

\subsection{Results and Discussion}

$T_{0}$ was obtained by processing the 268 images and divided into five intervals. One image was selected from each interval for the manual threshold, and the results are shown in Fig.4. Fig.4-a is a Near-infrared original image, Fig.4-b is a threshold binary image of $T_{0}$ and Fig.4-c is the binary image of $T_{1}$, Fig.4-d is the binary image of Model Threshold $T$.

(1) The image processing results are shown in Fig.4 which mainly include flaws and scratch. This processing the reflection of light effectively and simplify the image processing based on Near-infrared light to detect surface defects of kiwifruit. Using $P$ and interval of $T_{0}$ could detect the defects of fruit. There was some difference in the extent of noise influence. Results showed that interval of $T_{0}$ value from 0.1 to 0.2 had better effects than those of other intervals. Thus mathematical morphological operation was used to remove the noises.

(2) Using $T_{0}$ alone does not detect the surface defects for kiwifruit from Near-infrared image; however, it provided the precondition to quantify the standard of image segmentation reasonably, which meant that we could process different brightness images under the same standard. In Fig.5, for example, the binary images of $T_{0}$ were almost the same though different intervals were selected.

(3) Compared with the results of image processing in [18], the results in this paper were much better as it overcame the influence of fruit calyx.

(4) Fig.4-a is Near-infrared original image. Fig.4-d is binary image of $T$. Fig.4-c is binary image of $T_{l}$. When two images were compared, both detected the fruit surface defects. Therefore to make further comparison, the pixel values of the two binary images were calculated. As for Tab.5, Where: $p_{\mathrm{i} 1}$ is pixel value of Fig.4-d and $p_{i 2}$ is pixel value of Fig.4-c.

Table 5. The pixel values $p_{i j}$ for surface defects extract threshold

\begin{tabular}{ccccc}
\hline$T_{0}$ & $T\left(T=T_{0}+P\right)$ & $T_{1}$ & $p_{i 1}$ & $p_{i 2}$ \\
\hline 0.0784 & 0.1184 & 0.1180 & 2789 & 2789 \\
0.1490 & 0.2490 & 0.2450 & 1458 & 2214 \\
0.2745 & 0.4345 & 0.4305 & 7275 & 5521 \\
0.3765 & 0.5965 & 0.6050 & 7022 & 8459 \\
0.4039 & 0.6839 & 0.7000 & 3716 & 5450 \\
\hline
\end{tabular}




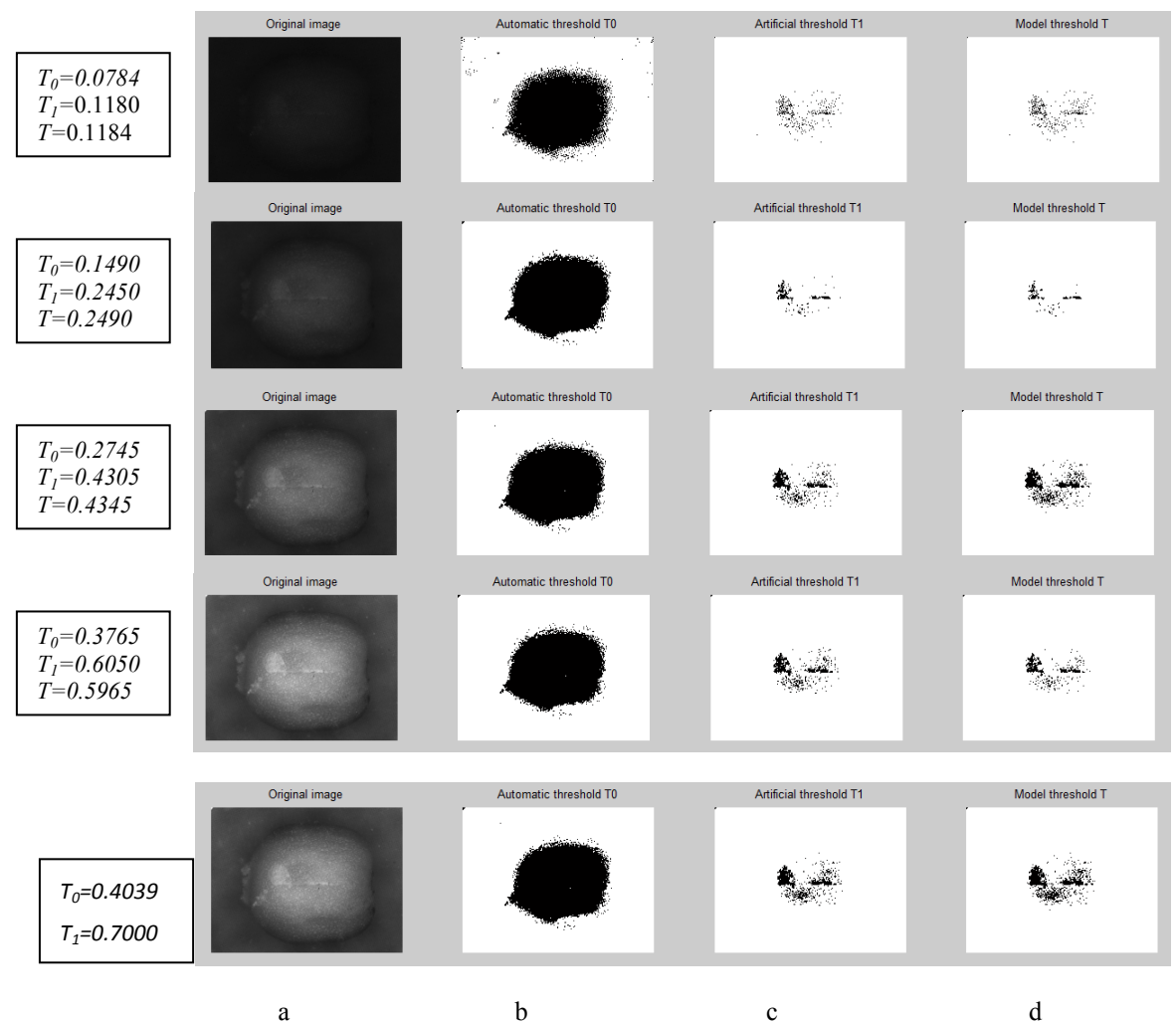

Fig. 4. Five image processing results

\section{Conclusion}

The function of Near-infrared light intensity and automatic threshold showed obvious linear relationship, with function fitting coefficient of $R^{2}>95 \%$. A linear mathematical model was developed to detect threshold of kiwifruit surface defects automatically.

In the five intervals of $T_{0}$, the Near-infrared image processing excluded the interference of the fruit calyx and therefore this method was more effective to detect fruit defects of surface flaws and scratches, especially with $T_{0}$ range of 0.1 to 0.2.

Overall our data provide a promising tool for automatic defects detection in the fruit grading system.

Acknowledgments. This research was funded by The Project-sponsored by SRF for ROCS, SEM (KS08021101), Project supported by the National Natural Science Foundation of China (61175099) and Northwest Agriculture and Forestry University Talent Fund (Z111020902). 


\section{References}

1. Rashidi, M., Seyfi, K.: Classification of Fruit Shape in Kiwifruit Applying the Analysis of Outer Dimensions. Int. J. Agric.Biol. 5, 759-762 (2007)

2. Tao, Y., Wen, Z.: An Adaptive Image Transform for High-speed Fruit Defect Detection. Transactions of the ASAE 42(1), 241-246 (1999)

3. Feng, B., Wang, M.: Study on Identifying Measurement about Default of Fruit in Computer Vision. Journal of China Agricultural University 7(4), 73-76 (2002)

4. Fu, F., Ying, Y.: Gray Level Transform Model of Ball Image and Its Application in Citrus Image Correction. Transactions of the Chinese Society of Agricultural Engineering 20(4), 117-120 (2004)

5. Ying, Y., Fu, F.: Color Transformation Model of Fruit Image in Process of Non-destructive Quality Inspection Based on Machine Vision. Transactions of the Chinese Society for Agricultural Machinery 35(1), 85-89 (2004)

6. Cheng, F., Ying, Y.: Inspection of Mildewed Rice Seeds Based on Color Feature. Transactions of the CSAE 35(4), 102-105 (2002)

7. Zhu, W., Cao, Q.: Defect Segmentation of Tomatoes Using Fuzzy Color Clustering Method. Transactions of the Chinese Society of Agricultural Engineering 19(3), 133-136 (2003)

8. Pang, J.: Study on External Defects Classification of Navel Orange Based on Machine Vision. Zhenjiang University, Hangzhou (2006)

9. Yang, F., Zhu, S., Qiu, Q.: Prickly Ash Appearance Quality Detection Based on Computer Vision and its Implementation in MATLAB. Transactions of the CSAE 24(1), 198-202 (2008)

10. Diaz, R., Gil, L., Serrano, C., Blasco, M., Moltó, E., Blasco, J.: Comparison of Three Algorithms in the Classification of Table Olives by Means of Computer Vision. Journal of Food Engineering 61(1), 101-107 (2004)

11. Blasco, J., Aleixos, N., Moltó, E.: Computer Vision Detection of Peel Defects in Citrus by Means of a Region Oriented Segmentation Algorithm. Journal of Food Engineering 81(3), 384-393 (2007)

12. Nagata, M., Jasper, G.T., Taiichi, K., Cui, Y., Yoshinori, G.: Predicting Maturity Quality Parameters of Strawberries Using Hyperspectral Imaging. In: ASAE/CSAE Annual International Meeting, 2004 (2004)

13. Nagata, M., Jasper, G.T., Taiichi, K., Hiroshi, T.: NIR Hyperspectral Imaging for Measurement of Internal Quality in Strawberries. In: ASABE Annual International Meeting 2005 (2005)

14. Jasper, G.T., Nagata, M., Taiichi, K.: Detection of Bruises in Strawberies by Hyperspectral Imaging. In: ASABE Annual International Meeting 2006 (2006)

15. Zhan, H., Li, X., Wang, W., Wang, C., Zhou, Z., Huang, Y.: Determination of Chestnuts Grading Based on Machine Vision. Transactions of the CSAE 26(4), 327-331 (2010)

16. Wen, Z., Tao, Y.: Dual-camera NIR/MIR Imaging for Stem-end/Calyx Identification in Apple Detect Sorting. Transactions of the ASAE 43(2), 449-452 (2000)

17. Li, J., Xue, L.: A Study on Navel Orange Grading System Based on Computer Vision. Jiangxi Agricultural University 28(2), 304-307 (2006)

18. Li, P., Ding, X., Su, S., Cui, Y.: A Method for Surface Defects Detection in Kiwi Fruit Classification. CSAE (2011)

19. Li, Q., Wang, M.: A Fast Identification Method for Fruit Surface Defect Based on Fractal Characters. Journal of Image and Graphics 5(2), 144-148 (2000) 